\title{
¿Qué hacen los estudiantes mientras planean una tarea? Los efectos de diferentes tiempos en una tarea oral
}

What do students do while planning a task? The effects of different time lengths in an oral task

Miguel Ángel Novella Gómez Eastern Washington University, Modern Languages Department

\section{Resumen}

Este estudio analiza cuantitativamente los efectos de tres tiempos diferentes de planeación en la fluidez, la complejidad y la precisión en una tarea de producción oral. Asimismo, se buscó explorar cualitativamente la asignación de los recursos lingüísticos en esa misma tarea mediante un cuestionario. Los 38 estudiantes universitarios de español que participaron en el estudio fueron asignados aleatoriamente a alguna de las tres condiciones de tiempo. Un diseño de $2 \times 3$ consideró dos niveles de proficiencia (intermedio y avanzado) y tres condiciones de planeación $(0,2$ y 5 minutos). Cada muestra fue transcrita y analizada de acuerdo con cuatro varia- bles dependientes: duración de la pausa, velocidad del habla, cantidad de subordinación y porcentaje de morfología verbal correcta. Las hipótesis, que predecían que el tiempo de planeación y nivel de proficiencia afectarían positivamente el desempeño de la tarea, fueron confirmadas de forma general. Los planeadores actuaron mejor que los no planeadores en velocidad del habla, cantidad de subordinación y porcentaje de morfología verbal correcta; el tiempo que permitió mostrar mejores resultados fue de cinco minutos. En cuanto al nivel de proficiencia, de forma general, los participantes avanzados se beneficiaron más de la planeación.

Palabras clave: enfoque por tareas; complejidad; precisión y fluidez; tiempo de planeación; tarea oral; español como segunda lengua 


\section{Abstract}

The aim of this study is twofold. First, to four dependent variables: pause length, it quantitatively analyzes the effects of three different planning lengths on fluency, complexity, and accuracy in an oral production task. Second, through a questionnaire, it qualitatively explores the allocation of linguistic resources in the same task. A total of 38 Spanish college students were randomized to one of 3 time conditions. A $2 \times 3$ study design considered two proficiency levels (intermediate and advanced) and three planning conditions (0, 2, 5 minutes). Each sample was transcribed and analyzed according speech rate, amount of subordination, and percentage of on-target verbal morphology. The hypotheses, which predicted that planning time and proficiency level would positively affect task performance, were confirmed in general terms. Planners performed better than non-planners in speech rate, amount of subordination, and percentage of on-target verbal morphology. To note, 5-minutes planning yielded better results. As regards overall proficiency level, advanced-level students benefited the most from planning.

Keywords: task-based language teaching; complexity; accuracy and fluency; planning time; oral task; Spanish as a second language 


\section{Introducción}

El acto de hablar una lengua es la prueba sine qua non de la proficiencia. Si bien expresarse en la lengua materna es un proceso automático y simple, hacerlo en una L2 resulta ser un proceso complejo que requiere mayor esfuerzo. De acuerdo al modelo teórico de Levelt $(1989,1993,1999)$, el proceso de producción en la L1 consta de tres etapas: el conceptualizador (conceptualizer), el formulador (formulator) y el articulador (articulator). En el caso de la L2, por otro lado, los hablantes enfrentan además cuatro fuentes de problemas: los déficits de recursos, la presión de tiempo en el procesamiento, las deficiencias percibidas en la producción de la lengua y las deficiencias percibidas en decodificar el mensaje del interlocutor (Dörnyei \& Scott, 1997).

De todos estos escollos, la presión que impone el procesar la información de forma rápida es uno de los obstáculos más acuciantes que afronta el hablante de segundas lenguas. Por ello, se puede decir que una de las diferencias cruciales entre la producción en L1 y L2 es que en la primera el procesamiento es automático, mientras que en la segunda se trata de un proceso controlado que requiere planeación. Ellis (1987) y Crookes (1989) aportaron los estudios seminales que analizaron los efectos de la planeación en la producción del lenguaje. A partir de estos estudios pioneros, el tiempo que se da para planear una tarea se ha convertido en una línea muy fructífera de investigación dentro de la instrucción por tareas (Crookes, 1989; Ellis, 2005; Foster \& Skehan, 1996; Sangarun, 2005; Wigglesworth \& Elder, 2010, entre otros).

Ofrecerle a un estudiante la oportunidad de planear repercute directamente en la manera en la que se completa la tarea (Ellis, 2005). La planeación en el enfoque por tareas ha sido dividida en dos rubros: a) la planeación antes de la tarea y b) la planeación durante la tarea. La distinción se refiere al momento en el que se lleva a cabo la planeación, ya sea antes o después de que la tarea ha sido iniciada. Además, los investigadores se han enfocado en estudiar las diferencias entre aquellas situaciones en las que los estudiantes completan la tarea por sus propios medios frente a aquellas en las que se les comunica con anticipación en qué deben enfocarse. En este último caso, se ha explorado la posibilidad de guiar a los estudiantes para que lleven a cabo conexiones entre el significado (es decir, el mensaje que se quiere transmitir), la forma (es de- 
cir, la gramática necesaria para transmitir ese mensaje) o entre el significado y la forma (Genc, 2012; Ortega, 2005; Sangarun, 2005).

\section{Estudios relacionados: el tiempo de planeación}

Crookes (1989) sugirió que la producción oral o escrita planeada empuja hacia el límite el interlenguaje y, consecuentemente, favorece el procesamiento de adquisición de una segunda lengua. En el mismo ámbito, Skehan (1996) desarrolló un marco teórico para el enfoque por tareas que incorpora a la planeación como una condición manipulable que regula la carga cognitiva, lo que permite a los estudiantes reducir la presión de la limitada memoria de trabajo para dirigir su atención hacia la forma y evitar así la propensión a enfocarse prioritariamente en el significado (VanPatten, 2004). Esto tiene consecuencias en tres áreas: fluidez, complejidad y precisión. La planeación promueve la fluidez al reducir el estrés comunicativo, la complejidad al facilitar la atención consciente hacia la forma y la precisión al permitir que los aprendices accedan a su máximo nivel gramatical y léxico (Housen, Kuiken \& Vedder, 2012; Muranoi, 2007).

\subsection{Los efectos de la planeación estratégica en la fluidez}

Crookes (1989), Foster y Skehan (1996), Kawauchi (2005), Mehnert (1998), Ortega (1999), Sangarun (2005), Wigglesworth y Elder (2010) y Yuan y Ellis (2003) han demostrado que la planeación estratégica se correlaciona positivamente con la fluidez.

Crookes (1989) encontró que, como se predecía, el grupo de planeación (10 minutos) produjo significativamente más que el grupo que no planeó en ambas tareas usadas en el estudio - medido en términos de palabras por emisión. Ortega (1999) halló mayor velocidad en una tarea que consistía en contar un cuento cuando los estudiantes de español de L2 tuvieron la oportunidad de planear con anticipación. Foster (2001), en un estudio que comparaba hablantes nativos y no nativos, reportó que los no nativos produjeron más al darles tiempo para planear, mientras que los nativo hablantes produjeron menos.

Sangarun (2005) examinó los diferentes efectos de dirigir la atención, ya sea en la forma, el significado o la forma y el significado, según dos condiciones 
de planeación: los que no planearon y los que lo hicieron por quince minutos. Los resultados para la fluidez revelaron que el tiempo para planear producía mayor fluidez en el habla.

\subsection{Los efectos de la planeación estratégica en la complejidad sintáctica}

Si bien los diferentes métodos de medición empleados hacen difícil comparar los resultados, Crookes (1989), Foster (2001), Kawauchi (2005), Mehnert (1998), Ortega (1999), Sangarun (2005), Skehan y Foster (1997) y Yuan y Ellis (2003) encontraron que el tiempo de la planeación tenía un efecto positivo en la complejidad sintáctica.

Yuan y Ellis (2003) exploraron los diferentes efectos de no planear, planear antes de la tarea y planear en el momento, y descubrieron que para los dos grupos que planearon había una correlación positiva entre planeación y complejidad sintáctica. Sin embargo, el grupo que planeó en el momento presentó un efecto estadístico significativo comparado con el grupo que no planeó. Crookes (1989) concluyó que planear por diez minutos produjo más oraciones complejas y un rango mayor de léxico. Foster y Skehan (1996) descubrieron que los que planearon con mayor detalle usaron considerablemente más subordinación que los planeadores no detallados, quienes a su vez produjeron más subordinación que los no planeadores. Según los investigadores, el tiempo para planear, ya sea de forma detallada o no, fomenta el uso de la subordinación y, por lo tanto, promueve una mayor complejidad sintáctica.

\subsection{Los efectos de la planeación estratégica en la precisión}

Sin embargo, los efectos de la planeación en la precisión son contradictorios. Crookes (1989) no encontró efectos en el caso de los artículos gramaticales al igual que Genc (2012), quien descubrió que el tiempo para planear no tuvo resultados en la precisión medida en porcentaje de cláusulas libres de error y porcentaje de formas verbales correctas. Ellis (1987), por su parte, en un estudio del uso del pretérito en inglés, concluyó que la planeación tuvo un efecto positivo en la precisión del pretérito regular, pero no en el caso del pretérito irregular. 
Wigglesworth (1997) condujo un estudio centrado en los efectos de la planeación en una situación de examinación. El investigador no descubrió ninguna diferencia estadísticamente significativa en el caso de los plurales y la morfología verbal entre un grupo al que se le dio tiempo para planear y un grupo al que no. Yuan y Ellis (2003), por su parte, apuntaron que el tiempo para planear no tuvo ningún efecto en la precisión.

Ortega (1999) reportó efectos mixtos. Según los resultados, el tiempo para planear tuvo un efecto positivo en los adjetivos, pero no en el caso de los artículos. Planteó que la precisión puede verse afectada de acuerdo con ciertas condiciones: a) la naturaleza de la planeación, según la cual la planeación no guiada favorece la precisión, b) la dificultad de la tarea, en la que una tarea fácil predispone la atención a la precisión, c) la duración del tiempo para planear, ya que un tiempo limitado para planear predispone una mayor atención a la precisión, y d) el nivel de proficiencia de los aprendices, puesto que los aprendices con un nivel alto de proficiencia se benefician más de las oportunidades para planear.

\subsection{Los efectos de los diferentes tiempos para planear}

Hay un número reducido de estudios que han explorado el tiempo como una variable en la producción oral, es decir, no solo comparan grupos de planeadores y no planeadores, sino también grupos que planean mientras realizan una tarea y, por lo tanto, estudian los efectos de los diferentes tiempos de planeación. Mehnert (1998) decidió indagar los efectos en un grupo que no planeó y tres diferentes tiempos para planear: uno, cinco y diez minutos. Con relación a la fluidez, se descubrió que, a pesar de observarse mayor fluidez a mayor cantidad de tiempo, la diferencia significativa se dio entre los planeadores y los no planeadores, por lo que más tiempo no incrementaba necesariamente la fluidez. Con relación a la precisión, la diferencia significativa ocurrió entre los que planearon un minuto y los que no planearon. Los que planearon cinco y diez minutos presentaron una precisión similar a la observada en los que no planearon. Por último, con respecto a la complejidad sintáctica, el grupo que presentó un efecto positivo fue el correspondiente a diez minutos. Los que planearon uno y cinco minutos presentaron una actuación similar a la observada por los que no planearon. Como resultado, según este estudio, algunos aspectos pueden bene- 
ficiarse con un tiempo tan limitado como un minuto de tiempo de planeación (fluidez y precisión), mientras que otros requieren más tiempo (complejidad).

\section{El presente estudio}

A pesar de que se han estudiado los efectos de la planeación con diversos propósitos, según lo revisado en la literatura, se han analizado tangencialmente los efectos de diferentes tiempos de planeación en dos niveles de proficiencia. Asimismo, se ha observado la falta de análisis de la planeación desde una perspectiva cualitativa, puesto que han prevalecido los del tipo cuantitativo. Para subsanar esta deficiencia, y mediante un análisis que permite la triangulación de datos, el propósito de este estudio consta de una vertiente cuantitativa y otra cualitativa. En el aspecto cuantitativo, explora los diferentes efectos del tiempo de planeación ( 0,2 y 5 minutos) en la fluidez, la complejidad sintáctica y la precisión en dos niveles de proficiencia (intermedio y avanzado). Con relación al aspecto cualitativo, investiga la actuación de los participantes mientras planean la tarea oral, y se une al puñado de estudios que han llevado a cabo este tipo de investigación (Genc, 2012; Ortega, 1999; Pang \& Skehan, 2014). El tiempo propuesto en este estudio es menor al que usaron Mehnert (1998) y Genc (2012), de diez minutos, o al de Sangarun (2005), de quince minutos, debido a que estudios previos han mostrado que incluso un tiempo de un minuto produce diferencias significativas en la producción de los participantes (Mehnert, 1998; Wigglesworth, 1997).

\section{Hipótesis}

Basadas en la revisión de la literatura, las hipótesis del estudio son las siguientes:

H1 Los planeadores actuarán mejor que los no planeadores.

H2 El tiempo de planeación se correlacionará significativamente con la fluidez y la complejidad sintáctica.

H3 Más tiempo para planear no impactará significativamente en la precisión.

H4 Los participantes avanzados se beneficiarán más del tiempo de planeación que los participantes intermedios. 


\section{Metodología}

\subsection{Participantes}

Los participantes fueron 38 estudiantes universitarios ( 23 mujeres y 15 hombres) inscritos en clases intensivas y no intensivas de español de niveles intermedio y avanzado en una universidad norteamericana. El rango de edad de los participantes fue de 18 a 22 años con un promedio de 19 años de edad. Las clases no intensivas constaban de sesiones de tres horas por semana, mientras que las clases intensivas consistían de cinco clases de una hora a la semana. En ambos casos los cursos eran comunicativos en los que se implementaba, en la medida de lo posible, el enfoque por tareas. Según el cuestionario de antecedentes, la mayoría de los participantes había estudiado español en un contexto formal, aunque cinco de ellos podrían ser clasificados como hablantes de herencia, ya que hablaban español en casa. Nueve de ellos habían estudiado español en algún país hispanohablante.

\subsection{La tarea}

La tarea oral consistía en narrar una historia en pretérito y en imperfecto a partir de 20 fotografías tomadas por el investigador. Las fotografías mostraban diferentes actividades de la vida estudiantil, por ejemplo, una persona haciendo su tarea, viendo la televisión, leyendo el periódico, revisando su correo electrónico, etcétera. Aunque las fotografías seguían un orden estructurado, se requería de la interpretación del narrador de la historia; es decir, las fotografías presentaban acciones que necesitaban cohesión y orden para formar una historia.

La tarea se seleccionó debido a que se centraba primordialmente en el significado, pues proveía un uso real de la lengua, fomentaba procesos cognitivos y tenía un producto definido (Ellis, 2003). Asimismo, la tarea no podía ser completada a menos que se usara la estructura deseada, por lo que cumplía el requisito de necesidad de la tarea (task-essentialness), según el concepto propuesto por Loschky y Bley-Vroman (1993).

Se buscó asegurar que la tarea fuera razonablemente demandante además de interesante, ya que las historias que requieren interpretación han probado ser 
una manera efectiva de forzar los recursos lingüísticos de los estudiantes, lo que las convierte en un medio apropiado para explorar los efectos de la planeación (Ortega, 1999; Skehan \& Foster, 1997, 1999). Las tareas narrativas, en lugar de las tareas interactivas, permiten medir con mayor precisión la producción de un estudiante, ya que en las segundas suele haber casos en los que alguno de los participantes no interviene y adopta una actitud pasiva. Además, las tareas interactivas son influenciadas por las intervenciones del interlocutor, como pueden ser sugerencias para clarificaciones, retroalimentaciones, confirmaciones, comentarios, etc., lo que agrega otra variable al estudio (Kawauchi, 2005).

\subsection{Diseño}

El estudio siguió un diseño $2 \times 3$ entre grupos con dos variables independientes: nivel de proficiencia (intermedio y avanzado) y tiempo para planear $(0,2$ y 5 minutos), y cuatro variables dependientes: duración de las pausas, velocidad del habla, cantidad de subordinación y porcentaje de morfología verbal correcta.

Los participantes fueron divididos aleatoriamente de acuerdo con las tres condiciones diferentes de tiempo de planeación, como se observa en el Cuadro 1. Los participantes que no tenían tiempo para planear recibieron una hoja con las instrucciones, se les mostraron las fotografías y se les pidió que comenzaran tan pronto como hubieran terminado de leer la tarea. Por el contrario, los sujetos que planearon (dos o cinco minutos) recibieron la hoja de la tarea con un párrafo que explicaba el tiempo con el que contaban para planear. Se les alentaba a que tomaran notas usando una hoja que se les proveyó para este propósito, pero se les advirtió que no podrían escribir oraciones completas o escribir todo con detalle. Después de concluido el tiempo para planear, los participantes tenían que entregar la hoja con sus anotaciones.

CuADRo 1. Número de participantes por condición de la tarea: nivel y tiempo de planeación

\begin{tabular}{lcccc}
\hline \multirow{2}{*}{ Nivel } & \multicolumn{3}{c}{ PLaneación ANTES DE LA TAREA } & \multirow{2}{*}{ N } \\
\cline { 2 - 4 } & 0 mins. & 2 mins. & 5 mins. & 21 \\
Intermedio & 9 & 6 & 6 & 17 \\
Avanzado & 5 & 7 & 5 & 38 \\
Total & 14 & 13 & 11 & 38 \\
\hline
\end{tabular}


Mientras veían las fotografías, los participantes debían narrar a un amigo, en este caso el investigador, las actividades que habían completado el día anterior. La intención de establecer una dinámica en la que una persona cuenta y la otra escucha fue construir una necesidad comunicativa para la tarea sin intervenir necesariamente en ella (Brown \& Yule, 1983; Ortega, 1999). Asimismo, y para para promover el uso del pretérito y el imperfecto, la oración en el tiempo pasado, “Ayer Juan...", se escribió en las instrucciones dadas a los estudiantes. La narración de cada participante fue grabada, transcrita y analizada en términos de fluidez, complejidad sintáctica y precisión.

\section{Análisis estadístico}

Las puntuaciones del investigador principal y otro evaluador, para $40 \%$ de las muestras, fueron analizadas con el fin de explorar la confiabilidad mediante el coeficiente alpha de Cronbach, el cual arrojó un resultado alto de confiabilidad de .963 en el caso de cantidad de subordinación y de .998 en el caso de la velocidad del habla. El resto de las muestras fueron codificadas por el investigador.

Las variables dependientes fueron analizadas según lo señalado por la unidad-AS (Foster, Tonkyn \& Wigglesworth, 2000). La unidad-AS toma como punto de partida la unidad-t, la cual considera las características de la lengua oral, tales como comienzos falsos, repeticiones, autocorrecciones, interrupciones, etc. La unidad-AS es la muestra del hablante que consiste en una cláusula independiente o unidad de subcláusula y cualquier cláusula subordinada relacionada con ella (Foster, Tonkyn \& Wigglesworth, 2000). Antes de conducir las ANOVAS para cada variable dependiente, se condujo una prueba de Leven para examinar la igualdad de las varianzas. Todos los análisis estadísticos en el estudio se llevaron a cabo usando el paquete estadístico sPss versión 21. Las transcripciones fueron analizadas según las siguientes variables dependientes:

- Fluidez

- Duración de las pausas

- Velocidad del habla 
- Complejidad sintáctica

- Cantidad de subordinación

- Precisión

- Porcentaje de morfología verbal correcta

\subsection{Medidas}

\subsubsection{Fluidez}

La fluidez se operacionalizó como duración de pausas y velocidad del habla (Mehnert, 1998). La duración de las pausas supone un parámetro para medir el grado en que los aprendices se detienen para planear su discurso oral, debido a que los estudiantes que pasan menos tiempo haciendo pausas han sido considerados como más fluidos (Ellis \& Barkhuizen, 2005). Se midió la duración de las pausas de un segundo o más. Este procedimiento se llevó a cabo dos veces para asegurarse de la precisión en la medición. No se hizo ninguna diferencia entre las pausas genuinas y las pausas que incluían expresiones de balbuceo como $\mathrm{mmm}, u \mathrm{hm}$, etc. El número representado fue la duración de las pausas.

Se calculó la velocidad del habla al determinar el número de sílabas cortadas por minuto. Las historias transcritas fueron cortadas para excluir las palabras autocorregidas, las repeticiones y las expresiones en la L1 (Ortega, 1999; Ellis \& Barkhuizen, 2005). El resultado total de sílabas cortadas se dividió posteriormente entre el número total en segundos que tomó producir la muestra multiplicada por sesenta. El resultado en sílabas por minuto fue la velocidad del habla.

\subsubsection{Complejidad sintáctica}

La cantidad de subordinación, o complejidad sintáctica, se obtuvo al dividir el número total de cláusulas entre el número de unidades-AS (Foster \& Skehan, 1999). En caso de que no hubiera ninguna subordinación en la muestra, la cantidad sería igual a 1 . 


\subsubsection{Precisión}

La precisión se midió al calcular el porcentaje de morfología verbal correcta (Wigglesworth, 1997). Se propone que la morfología correcta se correlaciona con la competencia general del aprendiz de L2 al suponer que un dominio de la flexión verbal refleja la capacidad de usar correctamente otras características gramaticales. Este porcentaje se obtiene al dividir el número de frases con verbos finitos correctos entre el número total de frases verbales multiplicadas por 100. Se analizaron los verbos en términos del tiempo, el aspecto, la modalidad y la concordancia entre el sujeto y el verbo.

\section{Resultados}

Las cuatro variables dependientes — duración de las pausas, velocidad del habla, cantidad de subordinación y porcentaje de morfología correcta- fueron analizadas a partir de una serie de ANOVAS factoriales para indagar las diferencias entre los distintos grupos de planeación y nivel de proficiencia. El nivel alpha fue ajustado a 0.05 y los exámenes post hoc Bonferroni se llevaron a cabo en aquellos casos en los que el valor de $p$ fue estadísticamente significativo $(p<.05)$. Se usaron exámenes de Bonferroni, ya que presentan mayor poder estadístico cuando el número de comparaciones es pequeño (Field, 2005). Los resultados de las ANOVAS factoriales se reportan en el Cuadro 2 de manera separada para fluidez [F] (duración de las pausas y velocidad del habla), complejidad [C] (cantidad de subordinación) y precisión [P] (porcentaje de morfología verbal correcta). El Cuadro 2 presenta los resultados de las estadísticas descriptivas para fluidez, complejidad y precisión y el Cuadro 3 presenta los resultados de las ANOVAS factoriales para fluidez, complejidad y precisión.

CuADro 2. Estadísticas descriptivas para fluidez, complejidad y precisión

\begin{tabular}{lll}
\hline \multicolumn{1}{c}{ VARIABLES } & \multicolumn{1}{c}{ INTERMEDIO } & AvanzADO \\
\hline [F] Duración de la pausa & & \\
No planeación & $43.77(11.91)$ & $10.2(4.6)$ \\
2 mins. & $24(9.44)$ & $14.14(8.07)$ \\
5 mins. & $23.5(6.47)$ & $8.6(2.97)$
\end{tabular}


Cuadro 2. Estadísticas descriptivas para fluidez, complejidad y precisión (continuación)

\begin{tabular}{lcc}
\hline \multicolumn{1}{c}{ VARIABLES } & INTERMEDIO & AvanZADO \\
\hline $\begin{array}{l}\text { [F] Velocidad del habla } \\
\text { No planeación }\end{array}$ & $76.23(18.7)$ & $116.14(18.5)$ \\
2 mins. & $104.2(27.7)$ & $120.1(24.1)$ \\
5 mins. & $106.6(18.9)$ & $139.1(15.3)$ \\
[c] Cantidad de subordinación & & \\
$\quad$ No planeación & $1.07(0.11)$ & $1.13(0.10)$ \\
2 mins. & $1.09(0.07)$ & $1.20(0.09)$ \\
5 mins. & $1.26(0.09)$ & $1.28(0.17)$ \\
[P] Porcentaje de morfología verbal & & \\
correcta & & \\
$\quad$ No planeación & & $66.87(16.18)$ \\
2 mins. & $63.92(15.11)$ & $81.40(11.98)$ \\
5 mins. & $44.69(22.52)$ & $92.38(4.64)$ \\
\hline
\end{tabular}

Clave: Media (Desviación estándar)

\subsection{Fluidez}

\subsubsection{Duración de las pausas}

Descriptivamente se puede mencionar que los estudiantes intermedios que no planearon $(M=43.77)$ presentaron una duración de las pausas mayor que la de los que planearon durante 2 minutos $(M=24)$, la cual a su vez es similar a la que presentó el grupo de 5 minutos $(M=23.5)$. Esto significó que para el nivel intermedio el tiempo para planear disminuyó las pausas e incrementó la fluidez conforme el tiempo de planeación se incrementó. En cambio, para el caso del nivel avanzado, se pudo observar que el grupo que no planeó $(M=10.2)$ mostró una duración de las pausas menor que el que planeó 2 minutos $(M=14.14)$. Resultó interesante constatar que la pausa para el grupo de 5 minutos resultó menor $(M=8.6)$ que la de los otros grupos, pero similar a la del grupo que no planeó (Cuadro 2).

En esta variable dependiente se encontraron efectos principales únicamente para el Grupo $(p<0.001)$, lo cual significa que la diferencia en porcentaje de 
duración de las pausas se explica por diferencias en la proficiencia; es decir, el nivel avanzado mostró una duración de la pausa menor que el intermedio. Sin embargo, no hubo un efecto para la Planeación $(p>.05)$ o para la interacción del Grupo $\times$ Planeación $(p>.05)$. Con respecto a los resultados significativamente no estadísticos para la planeación, se interpretó que no planear o planear 2 o 5 minutos no reduce la fluidez de forma estadísticamente significativa (Cuadro 3).

En cuanto al tiempo de planeación, se observó una variación, aunque no significativa, incluso con un tiempo breve de 2 minutos y, como resultado, más tiempo para planear (por ejemplo, 5 minutos) no redujo significativamente la duración de las pausas. Es decir, los grupos que planearon exhibieron un mejor promedio, pero con unos resultados que no son estadísticamente significativos. El orden del resultado fue $0=2=5$.

\subsubsection{Velocidad del habla}

Como se puede ver en el Cuadro 2, casi todas las medidas favorecieron a los grupos que planearon frente a los que no planearon. Descriptivamente, para el nivel intermedio, la condición de 5 minutos $(M=106.6)$ superó a la de 2 minutos $(M=104.2)$, sin embargo, las ganancias no fueron sustanciales. En comparación, el nivel avanzado presenta una velocidad del habla mucho mayor para la condición de 5 minutos $(M=139.1)$ que para la de 2 minutos $(M=120.1)$. Asimismo, como se puede ver, el nivel intermedio es el nivel de proficiencia que más se benefició de la Planeación, ya que pasó de una media de 76.23 para el grupo que no planeó frente a medias de 104.2 y 106.6 para los grupos de 2 y 5 minutos, respectivamente.

En este caso, hubo efectos significativos para el Grupo $(p<0.001)$ y la Planeación $(p=.014)$, mientras que no se presentaron efectos en la Interacción $(p>0.05)$. En cuanto a los resultados estadísticamente significativos para el Grupo, se interpreta que el nivel avanzado exhibió una velocidad del habla significativamente mayor a la del intermedio al presentar menos pausas. En el caso de los resultados para la Planeación, el examen post hoc Bonferroni reveló que no había una diferencia significativa entre los grupos que no planearon y los que lo hicieron durante 2 minutos, ni entre el grupo que no planeó y el grupo de 
5 minutos. La diferencia significativa fue únicamente entre los grupos de 2 minutos y el de 5 minutos. El orden presentado fue $0=2<5$ (Cuadro 3).

CuAdro 3. Resultados de ANOVAS factoriales para fluidez, complejidad y precisión

$\begin{array}{lllllllll}\text { MEDIDAS } & \text { SC } & G L & \text { MC } & F & P & \text { PARCIAL H } & \text { PODER }\end{array}$

[F] Duración de la pausa

$\begin{array}{lrrrrrrr}\text { Grupo } & 2025.00 & 1 & 1012.50 & 27.76 & <0.0001 & 0.594 & 1.0 \\ \text { Tiempo de planeación } & 181.99 & 1 & 90.99 & 2.49 & 0.096 & 0.116 & 0.47 \\ \text { Grupo x tiempo de planeación } & 197.62 & 2 & 49.4 & 4.65 & 0.268 & 0.125 & 0.381\end{array}$

[F] Velocidad del habla

$\begin{array}{lrrrrrrr}\text { Grupo } & 7899.5 & 1 & 7899.5 & 17.68 & <0.0001 & 0.356 & 0.983 \\ \text { Tiempo de planeación } & 4336.2 & 1 & 2168.1 & 4.85 & 0.014 & 0.233 & 0.762 \\ \text { Grupo x tiempo de planeación } & 970.02 & 2 & 485.1 & 1.086 & 0.350 & 0.064 & 0.223\end{array}$

[c] Cantidad de subordinación

$\begin{array}{llllllll}\text { Grupo } & 0.043 & 1 & 0.043 & 3.561 & 0.068 & 0.100 & 0.448 \\ \text { Tiempo de planeación } & 0.178 & 1 & 0.089 & 7.321 & 0.002 & 0.314 & 0.915 \\ \text { Grupo x tiempo de planeación } & 0.011 & 2 & 0.006 & 0.455 & 0.638 & 0.028 & 0.118\end{array}$

[P] Porcentaje de precisión verbal

$\begin{array}{llllllll}\text { Grupo } & 1889.49 & 1 & 1889.49 & 9.468 & 0.004 & 0.228 & 0.847 \\ \text { Tiempo de planeación } & 5374.255 & 1 & 2687.12 & 13.465 & <0.0001 & 0.457 & 0.996 \\ \text { Grupo x tiempo de planeación } & 2348.49 & 2 & 1174.57 & 5.883 & 0.007 & 0.269 & 0.842\end{array}$

\subsection{Complejidad}

\subsubsection{Cantidad de subordinación}

Se puede observar en el Cuadro 2 que los participantes del nivel intermedio mostraron un nivel superior de subordinación conforme tenían más tiempo de planeación, con una media de 1.07, 1.09 y 1.26 para el grupo que no planeó, el que planeó 2 minutos y el que planeó 5 minutos, respectivamente. Los estudiantes del nivel avanzado, por su parte, mejoraron según se incrementó el tiempo de 
planeación con unas medias de 1.13, 1.2 y 1.28, para el grupo que no planeó, el que planeó 2 minutos y el que planeó 5 , respectivamente.

No hubo un efecto significativo para el Grupo $(p>0.05)$ pero sí un efecto significativo para la Planeación $(p<0.001)$. El análisis no ofreció ninguna interacción significativa para el Grupo $\times$ Planeación, $F(2,32)=.455, p=0.638$. La prueba de post hoc mostró que no hubo diferencias entre el grupo de 0 minutos y el de 2 minutos $(p>.05)$; la comparación entre los 2 minutos y los 5 minutos fue significativa $(p<.05)$; así como la comparación entre los 0 minutos y los 5 minutos $(p<.01)$. En este caso la distinción entre los grupos operó entre más tiempo para planear y un tiempo corto para planear o ningún tiempo para planear. El orden fue $0=2<5$ (Cuadro 3 ).

\subsection{Precisión}

\subsubsection{Porcentaje de morfología verbal correcta}

Para el caso de los resultados de la variable de morfología verbal correcta se debe tener en cuenta que la prueba de Levene fue significativa $(p=.01)$, lo que implica que no se consiguió la asunción de la igualdad de las varianzas. Descriptivamente se puede ver en el Cuadro 2 que el nivel intermedio que no planeó $(M=63.92)$ mostró un porcentaje de precisión mayor que el grupo de 2 minutos $(M=44.69)$, el cual a su vez presentó un porcentaje menor que el de 5 minutos $(M=88.83)$. Por su parte, el nivel avanzado exhibió un porcentaje de precisión verbal mayor conforme se incrementaba el tiempo de planeación con una media de $66.87,81.4$ y 92.38 para el caso del grupo que no planeó y los grupos que planearon 2 y 5 minutos, respectivamente.

Los resultados de la ANOVA factorial para el porcentaje de morfología verbal correcta mostraron un efecto para el Grupo $(p<.01)$, para la Planeación $(p<.001)$ y para la interacción del Grupo $\times$ Planeación $(p<.01)$. Los resultados del post hoc evidenciaron que no existían diferencias significativas en el porcentaje de morfología verbal correcta entre el grupo de 0 minutos y el de 2 minutos $(p>.05)$. Sin embargo, se observaron diferencias significativas en la precisión entre el grupo de 0 minutos y el de 5 minutos y entre el de 2 minutos y el de 5 minutos $(p<.001)$. La puntuación siguió el orden $0=2<5$. Esto im- 
plica que se necesita más tiempo para observar los efectos de planeación en la precisión (Cuadro 3).

\subsection{Análisis cualitativo: cuestionario}

Con la finalidad de realizar un estudio exploratorio y cualitativo, después de completar la tarea, los participantes de los grupos que planearon contestaron de forma escrita un cuestionario diseñado para recopilar información acerca de su comportamiento durante el tiempo de planeación (Anexo). El cuestionario semiestructurado, elaborado a partir de unas preguntas hechas a dos participantes en una prueba piloto del experimento, buscaba analizar qué dicen los participantes respecto a lo que hacen durante el ejercicio. Las preguntas siguieron a la tarea de narración — se buscaba explorar la memoria de largo plazo (Gass \& Mackey, 2000). Con este propósito, las preguntas sondeaban si los participantes, mientras planeaban la tarea, se enfocaban en la gramática, el vocabulario, o ambos aspectos, y la escucha o los elementos retóricos y organizativos. Hay que tener presente que una práctica común de los docentes de lenguas es asignar cierto tiempo, si bien no tan controlado, para resolver una tarea o actividad.

CuAdro 4. Número de participantes centrados en diferentes elementos mientras planeaban la tarea

\begin{tabular}{cccccc}
\hline $\begin{array}{c}\text { Nivel/ } \\
\text { Tiempo de } \\
\text { planeación }\end{array}$ & $\begin{array}{c}\text { Centrado } \\
\text { en la } \\
\text { gramática }\end{array}$ & $\begin{array}{c}\text { Centrado } \\
\text { en el } \\
\text { vocabulario }\end{array}$ & $\begin{array}{c}\text { Centrado } \\
\text { en ambos } \\
\text { (gramática y } \\
\text { vocabulario) }\end{array}$ & $\begin{array}{c}\text { Centrado } \\
\text { en los aspectos } \\
\text { retóricos }\end{array}$ & $\begin{array}{c}\text { Consideran a } \\
\text { la persona } \\
\text { que escucha }\end{array}$ \\
\hline $\begin{array}{c}\text { Intermedio } \\
2 \text { minutos }\end{array}$ & 3 & 2 & 1 & 2 & 1 \\
5 minutos & - & - & 2 & & - \\
Avanzados & 2 & 3 & - & 1 & - \\
2 minutos & 1 & 2 & - & 3 & - \\
5 minutos & 2 & & & & \\
\hline
\end{tabular}

De forma general, según se puede observar en el Cuadro 4, y como se esperaba, la mayoría de los participantes se centraron en los aspectos lingüísticos (vocabulario o gramática), pero aun así es relevante que una tercera parte de ellos se enfocó en los aspectos retóricos de la historia, esto es, en establecer conexio- 
nes entre las fotografías para organizar la narración, tratar de entender de qué se trataban las fotografías e, incluso, tener en cuenta a la persona que escuchaba la narración; es decir, estos participantes realizaron la tarea como un acto comunicativo, por lo que se dedicaron a narrar una historia. Un número menor de participantes reportó enfocarse en la gramática y el vocabulario al mismo tiempo.

El nivel Intermedio de 2 minutos de planeación se enfocó en más aspectos (la gramática, el vocabulario, aspectos retóricos y gramática y vocabulario) que los grupos de nivel avanzado de 2 minutos y de 5 minutos, quienes se centraron en la gramática, el vocabulario y los aspectos retóricos. Sin embargo, se puede decir que el tener más tiempo para planear permite a los estudiantes dirigir la atención a los dos aspectos de la tarea: forma y significado, e incluso considerar a la persona que los escucha, mientras que un tiempo menor de duración limita su atención a solo un aspecto de la tarea, en este caso, los aspectos retóricos, como la gramática o el significado.

En cuanto al análisis cualitativo, según el nivel de proficiencia, se observa que los alumnos de nivel intermedio y nivel avanzado se enfocaban por igual en los aspectos gramaticales, en el vocabulario o en ambos, con un porcentaje de $66.66 \%$ u ocho de doce menciones.

Otras preguntas del cuestionario exploraban si el tiempo de planeación había sido útil para organizar la tarea y el modo de hacerlo, si la tarea había sido útil y si el tiempo otorgado para completar la tarea había sido suficiente. Cinco participantes mencionaron que se sintieron presionados mientras usaban el español en situaciones reales o, como mencionó uno de ellos, en "ser el centro de atención". Esta ansiedad mencionada por los participantes pudo incrementarse por el hecho de que cada estudiante realizaba la tarea con el investigador frente a una grabadora, lo cual crearía un ambiente en el que algunos participantes se sintieran examinados. A todo esto, hay que agregar que las tareas orales suelen crear ansiedad (Hewitt \& Stephenson, 2012). Tres de los participantes que planearon durante dos minutos consideraron que el tiempo dado era insuficiente, lo cual concuerda con los resultados obtenidos al observarse únicamente efectos estadísticamente significativos en el grupo que planeó durante cinco minutos. El grupo entero de los participantes que planearon encontró que el tiempo fue útil para emprender la tarea, sin embargo, dos participantes comentaron que el uso de un diccionario o el acceso a internet habría sido de más ayuda que únicamente el tiempo. 


\section{Discusión}

Los resultados apoyaron parcialmente las hipótesis. La hipótesis 1, que asumía que los planeadores como grupo actuarían mejor que los no planeadores, fue aprobada en parte, ya que los planeadores obtuvieron resultados estadísticamente significativos en tres de las cuatro variables estudiadas — velocidad del habla, cantidad de subordinación y porcentaje de morfología verbal correcta-, pero únicamente para el caso del grupo que planeó cinco minutos. Esto es, mientras que los no planeadores obtuvieron resultados estadísticamente similares a los del grupo que planeó dos minutos, los que planearon cinco minutos exhibieron una actuación que resultó estadísticamente más significativa. Esto parece demostrar que, si bien la planeación mejora la actuación, esta no presenta ningún efecto cuando se reduce el tiempo, por lo que el grupo de dos minutos actuó de forma similar al grupo que no planeó. De ahí que los grupos no se contrastaran entre planeadores y no planeadores, sino entre el grupo que no planeó y el de dos minutos frente al que planeó cinco minutos y exhibió resultados estadísticamente más significativos.

En el caso de la hipótesis 2, la cual señalaba que el tiempo de planeación se correlacionaría significativamente con la fluidez y la complejidad, esto es, que ambas se incrementarían conforme aumentara el tiempo de planeación, se observó que la correlación positiva se da únicamente a partir de la planeación de cinco minutos con la complejidad y con solo una de las variables en las que se midió la fluidez, la velocidad del habla. La hipótesis 3 predecía que el tiempo de planeación no influiría en la complejidad, y los resultados fueron similares a los presentados en las otras variables, donde cinco minutos produjeron efectos estadísticamente significativos, pero no así dos minutos. Finalmente, con relación a la hipótesis 4, que predecía que los participantes avanzados se beneficiarían más del tiempo de planeación que los participantes intermedios, el estudio demostró que, en tres de las cuatro variables estudiadas — fluidez (en las dos variables) y precisión, pero no así en complejidad- los niveles avanzados se beneficiaron mayormente del tiempo de planeación. Estos resultados apoyan la propuesta de Ortega (1999) que asume que el nivel mayor de proficiencia es aquel que se beneficia mayormente del tiempo para planear.

Los comentarios cualitativos de los participantes parecen apoyar los resultados de esta investigación, ya que tres de los estudiantes del tiempo de 2 minu- 
tos mencionaron que ellos creían que más tiempo para planear habría resultado en una mejor actuación.

En cuanto a los resultados no significativos de la duración de las pausas (fluidez), se observa que más tiempo no necesariamente significó una menor duración de las pausas, lo cual fue consistente con algunos estudios previos (Foster \& Skehan, 1996; Kawauchi, 2005; Ortega, 1999; Sangarun, 2005; Wigglesworth, 1997; Yuan \& Ellis, 2003).

Con relación a la complejidad, más tiempo para planear, en este caso cinco minutos, resultó en un nivel más alto de subordinación, lo cual es también consistente con estudios anteriores que señalan los efectos positivos en la complejidad conforme a mayor tiempo de planeación (Foster, 2001, Kawauchi, 2005, Sangarun, 2005). Por último, la precisión es un aspecto que acarrea una gran importancia en el área de planeación de la tarea, ya que se han obtenido resultados mixtos. De acuerdo con Ortega (1999), una tarea fácil predispone la atención hacia la precisión. Esta tarea — narrar una historia- es, por el contrario, demandante, de ahí que se pueda explicar por qué solamente después de cinco minutos, y no en dos minutos, se hayan obtenido resultados significativos con relación al porcentaje de morfología verbal correcta, que es como se midió la precisión en este estudio.

Sin embargo, al tiempo de planeación y al nivel de proficiencia debe agregarse otra variable: la complejidad de la tarea. La hipótesis de la cognición de Robinson (2001, 2003, 2005), por ejemplo, presupone que las tareas más complejas promueven más precisión y complejidad, aunque menos fluidez que las tareas simples. Al recordar que esta tarea es demandante, y tomando en cuenta las aseveraciones de los participantes — quienes señalaban que hubieran preferido tener más tiempo para planear-, se puede explicar por qué se necesitaron al menos 5 minutos para poder observar efectos positivos en la planeación en los tres ámbitos estudiados: complejidad, precisión y fluidez (únicamente en velocidad del habla).

\section{Conclusiones y limitaciones}

La manera en la que el tiempo de planeación influye en la precisión parece ser un problema todavía no resuelto en su totalidad. Si bien este trabajo ayuda a com- 
prender los efectos de la planeación, todavía hay aspectos que deben considerarse para tener un mejor entendimiento de cómo interactúan estos tres constructos.

Aunque estos resultados apoyan el supuesto de que los niveles avanzados se benefician más del tiempo de planeación (Ortega, 1999), es todavía poco claro cuánto tiempo se necesita para afectar la fluidez, la complejidad y la precisión. Una manera de corregir esta deficiencia es incluir el tipo de tarea como una variable (Ellis, 2009). El marco componencial triádico de Robinson (2007, 2011) es un prisma adecuado para interpretar los resultados según un marco teórico compartido. Según esta taxonomía, que a su vez abreva de la teoría de la cognición de Robinson (2001), las características de las tareas pedagógicas pueden ser clasificadas según tres componentes: 1) complejidad de la tarea, 2) condiciones de la tarea y 3) dificultad de la tarea. Contar con una categorización que reconozca las variables existentes en las distintas tareas permite hacer comparaciones entre estudios que puedan explicar las divergencias en los resultados. Si no se consideran las variables de la tarea, los estudios empíricos no podrán ser comparables, ya que la tarea en sí es una variable adicional a la investigación.

Una limitación que necesita ser atendida en este estudio es la inclusión de un examen que compruebe los niveles de proficiencia de los estudiantes, puesto que el único criterio para seleccionar a los participantes y clasificarlos en niveles fue el haber cursado estudios de nivel intermedio o avanzado. Aunque la institución aplicaba algún tipo de control para asegurarse de que los estudiantes estuvieran colocados en su nivel de proficiencia adecuado - tales como un examen de proficiencia solicitado por el alumno o una entrevista para saber con cuántos años de estudio contaba-, es un hecho que no todos los estudiantes tienen el mismo nivel de proficiencia a pesar de estar cursando un mismo grado. De ahí que dividir a los alumnos según el grado obtenido en una prueba de proficiencia es una manera óptima de subsanar esta deficiencia. Otras variables que deben ser tomadas en cuenta son las características individuales en la selección de los participantes, en este caso, el hecho de ser hablante de herencia. Es bien sabido que los hablantes de herencia presentan características muy particulares que los alejan de los hablantes tradicionales de español L2 (Montrul, 2012).

Si todas estas limitaciones son atendidas en estudios futuros, se podrá tener un mejor entendimiento de la forma en la que la planeación afecta la fluidez, la complejidad y la precisión. Pasaremos así de análisis predictivos a análisis ex- 
plicativos. Hay que recordar que el tiempo de planeación es una variable que los maestros de segundas lenguas suelen manipular, por lo que los resultados que arrojen este tipo de estudios son de interés tanto teórico como práctico.

\section{Referencias}

Brown, Gillian, \& Yule, George (1983). Teaching the spoken language: An approach based on the analysis of conversational English. Cambridge: Cambridge University Press.

Crookes, Graham (1989). Planning and interlanguage variation. Studies in Second Language Acquisition, 11(4), 367-383.

Dörnyei, Zoltán, \& Scott, Mary Lee (1997). Communication strategies in a second language: Definitions and taxonomies. Language Learning, 47(1), 173-210.

ElLIS, Rod (1987). Interlanguage variability in narrative discourse: Style shifting in the use of the past tense. Studies in Second Language Acquisition, 9(1), 1-19.

ElLIS, Rod (2003). Task-based language learning and teaching. Oxford: Oxford University Press.

Ellis, Rod (2005). Planning and task-based performance: Theory and research. En Rod Ellis (Ed.), Planning and task performance in a second language (pp. 3-36). Ámsterdam: John Benjamins.

ELLIS, RoD (2009). The differential effects of three types of task planning on the fluency, complexity, and accuracy in L2 oral production. Applied Linguistics, 30(4), 474-509. doi.org/10.1093/ applin/amp042

Ellis, Rod, \& Barkhuizen, Gary PAtrick (2005). Analyzing learner language. Oxford: Oxford University Press.

FIELD, ANDY (2005). Discovering statistics using SPSS. Londres: Sage.

Foster, Pauline (2001). Rules and routines: A consideration of their role in the task-based language production of native and non-native speakers. En Martin Bygate, Pete Skehan \& Merrill Swain (Eds.), Researching pedagogic tasks, second language learning, teaching and testing (pp. 75-94). Nueva York: Longman.

Foster, Pauline, \& Skehan, Peter (1996). The influence of planning and task type on second language performance. Studies in Second Language Acquisition, 18(3), 299-323.

Foster, Pauline, \& Skehan, Peter (1999). The influence of source of planning and focus of planning on task-based performance. Language Teaching Research, 3(3), 215-247.

Foster, Pauline; Tonkyn, Alan, \& Wigglesworth, Gillian (2000). Measuring spoken language: A unit for all reasons. Applied Linguistics, 21(3), 354-375. doi: 10.1093/ applin/21.3.354 
Gass, SusAn, \& MACKey, Alison (2000). Stimulated recall methodology in second language research. Mahwah: Lawrence Erlbaum.

Genc, Zubeyde Sinem (2012). Effects of strategic planning on the accuracy of oral and written tasks in the performance of Turkish EFL learners. En Ali Shehadeh \& Christine A. Coombe (Eds.), Task-based language teaching in foreign language contexts (pp. 67-88). Ámsterdam: John Benjamins.

Hewitt, Elaine, \& Stephenson, JeAn (2012). Foreign language anxiety and oral performance: A replication of Philips's “MLJ" study. Modern Language Journal, 96(2), 170-189. Recuperado de http://www.jstor.org/stable/41684068

Housen, Alex; Kuiken, Folkert, \& Vedder, Ineke (2012). Complexity, accuracy and fluency: Definitions, measurement and research. En Alex Housen, Folkert Kuiken \& Ineke Vedder (Eds.), Dimensions of L2 performance and proficiency. Complexity, accuracy and fluency in SLA (pp. 1-20). Ámsterdam: John Benjamins.

Kawauchi, Chieko (2005). The effects of strategic planning on the oral narratives of learners with low and high intermediate L2 proficiency. En Rod Ellis (Ed.), Planning and task performance in a second language (pp. 143-164). Ámsterdam: John Benjamins.

Levelt, Willem J. M. (1989). Speaking: From intention to articulation. Cambridge: Mit Press.

Levelt, Willem J. M. (1993). Language use in normal speakers and its disorders. En Gerhard Blanken, Jürgen Dittmann, Hannelore Grim, John C. Marshall \& Claus-W. Wallesch (Eds.), Linguistic disorders and pathologies: An International Handbook (pp. 1-15). Berlín: De Gruyter.

Levelt, Willem J. M. (1999). Producing spoken language: A blueprint of the speaker. En Colin M. Brown \& Peter Hagoort (Eds.), Neurocognitive of language (pp. 83-122). Oxford: Oxford University Press.

Loschiy, Lester, \& Bley-Vroman, Robert (1993). Grammar and task-based methodology. En Graham Crookes \& Susan M. Gass (Eds.), Tasks and language learning: Integrating theory and practice (pp. 123-167). Clevedon: Multilingual Matters.

Mehnert, Uta (1998). The effects of different lengths of time for planning on second language performance. Studies in Second Language Acquisition, 20(1), 52-83.

Montrul, SilvinA A. (2012). Is the heritage language like a second language? EuroSLA Yearbook, $12,1-29$.

Muranoi, Hitoshi (2007). Output practice in the L2 classroom. En Robert DeKeyser (Ed.), Practice in a second language: Perspectives from applied linguistics and cognitive psychology (pp. 51-84). Cambridge: Cambridge University Press. 
Ortega, Lourdes (1999). Planning and focus on form in L2 oral performance. Studies in Second Language Acquisition, 21(1), 109-148.

Ortega, Lourdes (2005). What do learners plan? Learner-driven attention to form during pretask planning. En Rod Ellis (Ed.), Planning and task performance in a second language (pp. 77-110). Ámsterdam: John Benjamins.

Pang, Francine, \& Skehan, Peter (2014). Self-reported planning behaviour and second language performance in narrative retelling. En Peter Skehan (Ed.), Processing perspectives on task performance (pp. 95-128). Ámsterdam: John Benjamins.

Robinson, Peter (2001). Task complexity, task difficulty, and task production: Exploring interactions in a componential framework. Applied Linguistics, 22(1), 27-57.

Robinson, Peter (2003). The Cognition Hypothesis, task design and adult task-based language learning. Second Language Studies, 21(2), 45-107.

Robinson, Peter (2005). Cognitive complexity and task sequencing: Studies in a componential framework for second language task design. International Review of Applied Linguistics in Language Teaching, 43(1), 1-32. doi.org/10.1515/iral.2005.43.1.1

Robinson, Peter (2007). Criteria for classifying and sequencing pedagogic tasks. En María del Pilar García Mayo (Ed.), Investigating tasks in formal language learning (pp. 7-27). Clevedon: Multilingual Matters.

Robinson, Peter (2011). Second language task complexity, the Cognition Hypothesis, language learning, and performance. En Peter Robinson (Ed.), Second language task complexity. Researching the Cognition Hypothesis of language learning and performance (pp. 3-38). Ámsterdam: John Benjamins.

SANGARUN, JiRAPORN (2005). The effects of focusing on meaning and form in strategic planning. En Rod Ellis (Ed.), Planning and task performance in a second language (pp. 111-142). Ámsterdam: John Benjamins.

Skehan, Peter (1996). A framework for the implementation of task-based instruction. Applied Linguistics, 17(1), 38-62. doi.org/10.1093/applin/17.1.38

Skehan, Peter, \& Foster, Pauline (1997). Task type and task processing conditions as influences on foreign language performance. Language Teaching Research, 1(3), 185-211.

Skehan, Peter, \& Foster, Pauline (1999). The influence of task structure and processing conditions on narrative retellings. Language Learning: A Journal of Research in Language Studies, 49(1), 93-120.

VanPatten, Bill (2004). Processing instruction: Theory, research, and commentary. Mahwah: Lawrence Erlbaum. 
Wigglesworth, Gillian (1997). An investigation of planning time and proficiency level on oral test discourse. Language Testing, 14(1), 85-106.

Wigglesworth, Gillian, \& Elder, Cathie (2010). An investigation of the effectiveness and validity of planning time in speaking test tasks. Language Assessment Quarterly, 7(1), 1-24. doi.org/10.1080/15434300903031779

Yuan, FAngyuan, \& Ellis, Rod (2003). The effects of pre-task and on-line planning on fluency, complexity and accuracy in L2 monologic oral production. Applied Lingustics, 24(1), 1-27. doi.org/10.1093/applin/24.1.1 


\section{Anexo}

Cuestionario estudio cualitativo: 2 minutos de planeación

Planeación

Nombre:

Nivel:

Fecha:

2 minutos de tiempo para planear

1) Durante el tiempo que tuviste para planear, ¿te preocupaste por la gramática, el vocabulario, ambos, la mejor manera de organizar la historia o la persona que estaba escuchando? Explica de la forma más detallada posible.

2) ¿Generalmente tomas algún tiempo para planear antes de usar la lengua meta?

3) ¿Crees que planear te ayuda a organizar la tarea? ¿De qué manera?

4) ¿Encontraste la actividad útil?

5) ¿Piensas que se te dio suficiente tiempo? Sí o no, por qué 